\title{
Pharmacotherapy Prescribing to Patients with Concurrent Tobacco and Alcohol Use Disorder in a Large, Urban, Integrated Health System
}

\author{
Mat Kladney, $\mathrm{MD}^{1,2}$, Paul Joudrey, $\mathrm{MD}, \mathrm{MPH}^{3,4}$, Chinazo O. Cunningham, $\mathrm{MD}, \mathrm{MS}^{7}$, and \\ Marcus A. Bachhuber, MD, MSHP
}

'Division of General Internal Medicine, Montefiore Medical Center/Albert Einstein College of Medicine, Bronx, NY, USA; ${ }^{2}$ Division of General Internal Medicine and Clinical Innovation, New York University - Bellevue Hospital, New York, NY, USA; ${ }^{3}$ VA Connecticut Healthcare System, West Haven Campus, West Haven, CT, USA; ${ }^{N}$ National Clinician Scholars Program, Yale School of Medicine, New Haven, CT, USA.

J Gen Intern Med 34(6):804-5

DOI: $10.1007 / \mathrm{s} 11606-018-4806-\mathrm{y}$

(c) Society of General Internal Medicine 2018

\section{INTRODUCTION}

Tobacco use disorder (TUD) and alcohol use disorder (AUD) are two leading causes of preventable death in the USA. These disorders frequently occur concurrently, leading to multiplicative effects on morbidity and mortality. ${ }^{1}$ Treatment of AUD and TUD with pharmacotherapy is an evidence-based way to reduce these harms. The prevalence of pharmacotherapy prescribing for patients with coexisting AUD and TUD is poorly understood. We conducted a retrospective cohort study to determine the prevalence of prescribing of AUD and TUD pharmacotherapy in a population with both diagnoses.

\section{METHODS}

We conducted a retrospective cohort study using electronic health record data from Montefiore Health System between January 2010 and December 2014. Montefiore Health System is a large urban academic medical center and integrated health care system in the Bronx, New York, with over 3 million outpatient visits annually at a network of almost 100 primary and specialty care clinics.

Inclusion criteria were (1) age $\geq 18$ years and (2) concurrent TUD and AUD diagnoses, defined by International Classification of Diseases, 9th Revision, Clinical Modification, diagnosis codes (ICD-9) or problem list entries indicating both TUD and AUD (list available from the authors upon request). We defined concurrent TUD and AUD as both disorders being actively documented during the study period and actively documented within 90 days of each other. We excluded patients if they were prescribed TUD or AUD pharmacotherapy within 6 months prior to cohort entry (defined as time of AUD diagnosis).

This data was first presented at the Association for Medical Education and Research in Substance Abuse National Conference in Washington DC in November 2016.

Published online January 2, 2019
We defined TUD pharmacotherapy as any prescription for nicotine replacement therapy, varenicline, or bupropion within 1 year of TUD diagnosis. We defined AUD pharmacotherapy as any prescription for acamprosate, naltrexone, topiramate, or disulfiram within 1 year of AUD diagnosis. Despite not being FDA-approved for treatment of AUD, we included topiramate as it is used off-label for this indication. In addition, we extracted age, sex, race/ethnicity, and insurance status.

To describe trends in pharmacotherapy prescribing, we classified patients into mutually exclusive categories: no pharmacotherapy, AUD pharmacotherapy only, TUD pharmacotherapy only, or both. For each year, we calculated the percentage of patients in each category. We used chi-square tests to assess the trend in the incidence of pharmacotherapy over the 5-year period.

Finally, we conducted a sensitivity analysis by excluding patients from the cohort who had alternative indications for the pharmacotherapies (major depressive disorder and buproprion, seizure disorder or migraine headaches and topiramate, and opioid use disorder and naltrexone).

The study was approved by the Albert Einstein College of Medicine Institutional Review Board.

\section{RESULTS}

We identified 5065 patients with concurrent TUD and AUD diagnoses. The cohort was $71.9 \%$ male, $40.7 \%$ non-Hispanic Black, and $71.2 \%$ publicly insured (Table 1). Over the 5-year study period, most patients $(87.7 \%)$ were not prescribed pharmacotherapy for TUD or AUD within one year of cohort entry. TUD pharmacotherapy alone was prescribed to $10.6 \%$, AUD pharmacotherapy alone was prescribed to $1.4 \%$, and both were prescribed to $0.3 \%$ of patients.

The percentage of patients prescribed pharmacotherapy increased during the study period for all groups: TUD pharmacotherapy only ( 9.5 to $14.2 \%, p<0.001)$, AUD pharmacotherapy only $(1.0$ to $2.1 \%, \mathrm{p}<0.001)$, and both TUD and AUD pharmacotherapy ( 0.5 to $0.7 \%, p=0.04$ Fig. 1 ). In our sensitivity analysis, estimates of TUD pharmacotherapy prescribing alone (10.4\%) and AUD pharmacotherapy prescribing alone $(1.1 \%)$ over the study period were slightly lower than our main analysis. 
Table 1 Demographic and clinical characteristics of patients with new, concurrent, alcohol, and tobacco use disorder diagnoses in a large urban integrated health care system $(n=\mathbf{5 0 6 5})$

\begin{tabular}{ll}
\hline \hline Characteristic & All patients $(\boldsymbol{n}=\mathbf{5 0 6 5})$ \\
\hline Age, $n(\%)$ & \\
$18-29$ & $615(12.1)$ \\
$30-44$ & $1334(26.3)$ \\
$45-64$ & $2518(49.7)$ \\
$65+$ & $598(11.8)$ \\
Female sex, $n(\%)$ & $1424(28.1)$ \\
Race/ethnicity, $n(\%)$ & $432(8.5)$ \\
Hispanic, of any race & $844(16.7)$ \\
Non-Hispanic white & $2044(40.4)$ \\
Non-Hispanic black & $1320(26.1)$ \\
Any other ${ }^{a}$ or undetermined race & \\
Insurance status, $n(\%)$ & $3604(71.2)$ \\
Public & $1145(22.6)$ \\
Private & $266(5.3)$ \\
Self-pay & $2380(47.0)$ \\
Charlson score, $n$ (\%) & $1697(33.5)$ \\
0 & $988(19.5)$ \\
$1-2$ &
\end{tabular}

\section{DISCUSSION}

At a large urban health care system, prescribing of TUD and AUD pharmacotherapy to patients with both disorders was

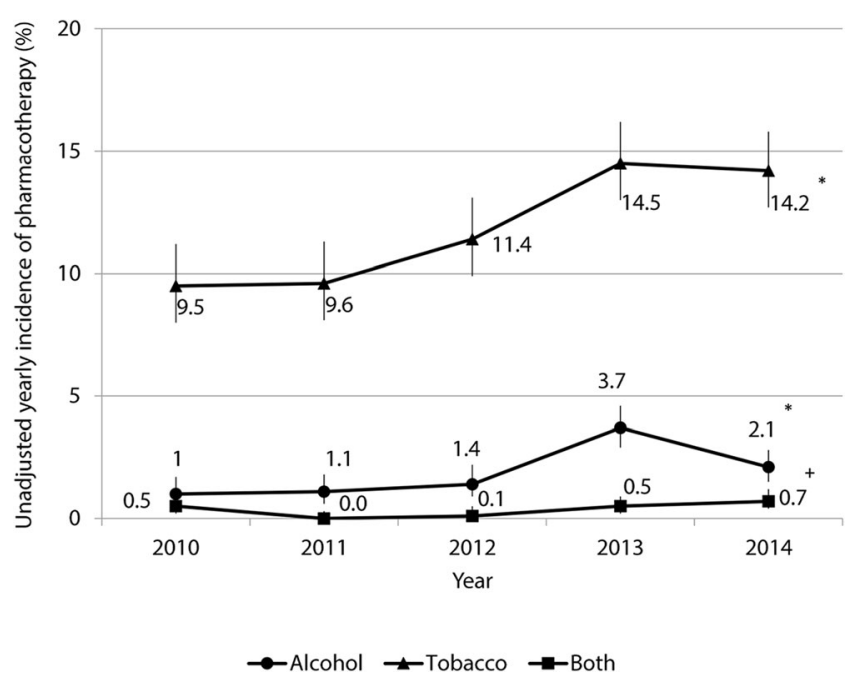

Fig. 1 Percentage of patients with new, concurrent, tobacco, and alcohol use disorder receiving a pharmacotherapy prescription in a large urban integrated health care system, 2010-2014. Error bars represent $95 \%$ CI. Alcohol pharmacotherapy represents the percentage of patients who received acamprosate, naltrexone, topiramate, or disulfiram. Tobacco pharmacotherapy represents the percentage of patients who received nicotine replacement therapy,

bupropion, or varenicline. Both includes patients who received pharmacotherapy for both alcohol and tobacco use. *Chi-square test $p<0.001,{ }^{+}$chi-square test $p=0.04$ exceptionally low. Although the rate of pharmacotherapy prescribing increased over the study period, we found prescribing rates were much lower than other studies assessing pharmacotherapy prescriptions for each diagnosis in isolation ${ }^{2,3}$ or with coexisting diagnoses. ${ }^{4}$ Because our analysis did not exclude patients with AUD or TUD diagnosis prior to the cohort period, we may underestimate the prevalence of pharmacotherapy prescribing.

Given our study setting and patient population, low prescribing rates may also reflect disparities in care among racial/ ethnic minorities and the urban poor, as seen in other studies. ${ }^{5}$ Over the last several decades, clinical, research, and policy interventions have increased the rates of TUD pharmacotherapy prescribing. ${ }^{6}$ Similar efforts are needed to identify and treat patients with concurrent substance use disorders, with a focus on addressing racial/ethnic disparities.

Corresponding Author: Mat Kladney, MD; Division of General Internal Medicine and Clinical Innovation, Bellevue Hospital, New York, NY, USA (e-mail: kladney@gmail.com).

Funding Information This study was funded in part by grant from the National Institute on Drug Abuse of the National Institutes of Health (K24DA036955 and K08DA043050). The funding agency had no role in design of the study, collection, analysis, and interpretation of data, or in writing the manuscript.

\section{Compliance with Ethical Standards:}

The study was approved by the Albert Einstein College of Medicine Institutional Review Board.

Conflict of Interest: The authors declare that they do not have a conflict of interest.

Publisher's Note: Springer Nature remains neutral with regard to jurisdictional claims in published maps and institutional affiliations.

\section{REFERENCES}

1. MacLean RR, Sofuoglu M, Rosenheck R. Tobacco and alcohol use disorders: evaluating multimorbidity. Addict Behav. 2018;78:59-66.

2. Harris AH, Oliva E, Bowe T, Humphreys KN, Kivlahan DR, Trafton JA. Pharmacotherapy of alcohol use disorders by the Veterans Health Administration: patterns of receipt and persistence. Psychiatr Serv. 2012;63(7):679-85.

3. Shiffman S, Brockwell SE, Pillitteri JL, Gitchell JG. Use of smokingcessation treatments in the United States. Am J Prev Med. 2008;34(2):102-11.

4. Rubinsky AD, Chen C, Batki SL, Williams EC, Harris AH. Comparative utilization of pharmacotherapy for alcohol use disorder and other psychiatric disorders among U.S. Veterans Health Administration patients with dual diagnoses. J Psychiatr Res. 2015;69:150-7.

5. Mennis J, Stahler GJ. Racial and Ethnic Disparities in Outpatient Substance Use Disorder Treatment Episode Completion for Different Substances. J Subst Abuse Treat. 2016;63:25-33.

6. Joseph AM, Willenbring ML, Nugent SM, Nelson DB. A randomized trial of concurrent versus delayed smoking intervention for patients in alcohol dependence treatment. J Stud Alcohol. 2004;65(6):681-91. 